\title{
pp interactions in extended air showers
}

\author{
A. Kendi Kohara ${ }^{\mathrm{a}}$, Erasmo Ferreira ${ }^{\mathrm{b}}$, and Takeshi Kodama ${ }^{\mathrm{c}}$ \\ Instituto de Física, Universidade Federal do Rio de Janeiro, CP 68528, Rio de Janeiro 21945-970, RJ, Brazil
}

\begin{abstract}
Applying the recently constructed analytic representation for the pp scattering amplitudes, we present a study of p-air cross sections, with comparison to the data from Extensive Air Shower (EAS) measurements. The amplitudes describe with precision all available accelerator data at ISR, SPS and LHC energies, and its theoretical basis, together with the very smooth energy dependence of parameters controlled by unitarity and dispersion relations, permit reliable extrapolation to higher energies and to asymptotic ranges. The comparison with cosmic ray data is very satisfactory in the whole pp energy interval from 1 to $100 \mathrm{TeV}$. High energy asymptotic behaviour of cross sections is investigated in view of the geometric scaling property of the amplitudes. The amplitudes predict that the proton does not behave as a black disk even at asymptotically high enegies, and we discuss possible non-trivial consequences of this fact for $\mathrm{pA}$ collision cross sections at higher energies.
\end{abstract}

\section{Introduction}

The data on pA collisions extracted from studies of Extensive Air Showers (EAS) reaches the $100 \mathrm{TeV}$ region of pp center of mass energies $(\sqrt{s})$ that are well above the present accelerator experiments. These data contain very important information on the dynamics of strong interactions at extreme high energies and also on the origin of high energy cosmic rays (CR). Thus the earth atmosphere is used as a detector of high energy particles to investigate the high energy phenomena in the Universe. On the other hand, different from the laboratory experiment, analysis of these data is more dependent on models that permit reliable extrapolation to this energy region.

Recent detailed analyses [1-5] of the experimental $\mathrm{pp}$ and $\mathrm{p} \overline{\mathrm{p}}$ scattering data based on a QCD inspired model $[6,7]$ reproduces the observed cross sections for all energies with high accuracy. These analyses lead to a precise analytic representation of the elastic scattering amplitudes, disentangling the real and imaginary parts, respecting unitarity and causality conditions, with regular energy dependence that gives confidence in extrapolation to higher energies. The purpose of the present work is to calculate the proton-air production cross section in the framework of the Glauber model using this representation of pp scattering as input, and compare the results to the experimental values obtained from the available cosmic ray $(\mathrm{CR})$ data. We are mainly concerned with the energies beyond the LHC experiments but also present results for EAS experiments in the region below $1 \mathrm{TeV}$.

Our analysis of energy dependence of amplitudes and observables in pp collisions shows that the total cross section has a neat $\log ^{2} s$ form [5], as already indicated in several analyses [8]. An important new feature is that the slope parameters, $B_{I}$ and $B_{R}$, also have a $\log ^{2} s$

\footnotetext{
a e-mail: kendi@if.ufrj.br

b e-mail: erasmo@if.ufrj.br

c e-mail: tkodama@if.ufrj.br
}

dependence. This is a new finding, since the generally accepted idea is that the slope of the differential cross sections varies like simple linear $\log s$, as in Regge phenomenology. This result has a crucial effect for the use of Glauber formalism in the analysis of p-air extended showers at the high energies of our concern, since the value of the slope $B_{I}$, together with the value of the total cross section, are the basic inputs of the calculation.

For the application of the standard Glauber approach, we basically need the amplitudes in forward scattering. In these conditions our amplitudes take simpler exponential forms requiring only two parameters to specify each amplitude. The relevant parameters are then the total cross section $\sigma$, the ratio $\rho$ between real and imaginary parts at $t=0$, and the slopes $B_{I}$ and $B_{R}$ of each of the two parts. Our full- $t$ analysis [5] provides the energy dependence of these quantities with simple analytical forms that are appropriate for the whole energy range from $50 \mathrm{GeV}$ to $100 \mathrm{TeV}$.

On the other hand, the $\log ^{2} s$ dependence of the slopes is intimately related to the unitarity condition, as can be seen more clearly in the $b$-space representation. Another interesting consequences of our representation is that a proton does not behave as a black disk even in asymptotic energy domain. This fact also has important consequences to the estimate of $\mathrm{pA}$ collision cross section in the ultrahigh energy domain.

\section{Forward scattering amplitudes}

In the treatment of elastic pp and $p \bar{p}$ scattering in the forward direction, with amplitudes approximated by pure exponential forms, the differential cross section is written

$$
\begin{aligned}
\frac{d \sigma}{d t}= & \pi(\hbar c)^{2}\left\{\left[\frac{\rho \sigma}{4 \pi(\hbar c)^{2}} e^{B_{R} t / 2}+F^{C}(t) \cos (\alpha \Phi)\right]^{2}\right. \\
& \left.+\left[\frac{\sigma}{4 \pi(\hbar c)^{2}} e^{B_{I} t / 2}+F^{C}(t) \sin (\alpha \Phi)\right]^{2}\right\}
\end{aligned}
$$

This is an Open Access article distributed under the terms of the Creative Commons Attribution License 4.0, which permits unrestricted use, distribution, and reproduction in any medium, provided the original work is properly cited. 
where $t \equiv-|t|$ and we must allow different values for the slopes $B_{I}$ and $B_{R}$ of the imaginary and real amplitudes. With $\sigma$ in milibarns and $|t|$ in $\mathrm{GeV}^{2}$, we have $(\hbar c)^{2}=0.3894$. Since we work with $B_{R} \neq B_{I}$, treatment of the Coulomb interference requires a more general expression for the Coulomb phase, which has been developed before [1]. However, in the present work we only need the forward $(|t|=0)$ nuclear amplitudes and slopes, and the Coulomb interaction does not enter, and we put $F^{C}(t)=0$.

The energy dependences of the four quantities are given by

$$
\begin{gathered}
\sigma(s)=69.3286+12.6800 \log \sqrt{s}+1.2273 \log ^{2} \sqrt{s} \\
B_{I}(s)=16.2472+1.5392 \log \sqrt{s}+0.17476 \log ^{2} \sqrt{s} \\
B_{R}(s)=22.835+2.862 \log \sqrt{s}+0.32972 \log ^{2} \sqrt{s}
\end{gathered}
$$

and

$$
\rho(s)=\frac{3.528018+0.785609 \log \sqrt{s}}{25.11358+4.59321 \log \sqrt{s}+0.444594 \log ^{2} \sqrt{s}},
$$

where $\sqrt{s}$ is in $\mathrm{TeV}, \sigma$ in milibarns, $B_{I}$ and $B_{R}$ are in $\mathrm{GeV}^{-2} ; \rho$ is dimensionless, passes through a maximum at about $1.8 \mathrm{TeV}$, and decreases at higher energies, with asymptotic value zero. The ratio $B_{R} / B_{I}$ is always larger than one, as expected from dispersion relations [9], and behaves asymptotically like

$$
\frac{B_{R}}{B_{I}} \rightarrow 1.8867-\frac{0.24065}{\log \sqrt{s}}-\frac{42.6214}{\log ^{2} \sqrt{s}} .
$$

The dimensionless ratio

$$
R_{I}=\frac{1}{(\hbar c)^{2}} \frac{\sigma}{16 \pi B_{I}}
$$

is important in the study of the form of the pp interaction. In our description of the pp system, as given by the energy dependences in Eqs. ((2)-(5)), this ratio has the high energy behaviour

$$
R_{I}=\frac{1}{(\hbar c)^{2}} \frac{\sigma}{16 \pi B_{I}} \rightarrow 0.3588+\frac{0.54673}{\log \sqrt{s}}-\frac{17.9064}{\log ^{2} \sqrt{s}}
$$

From the pure exponential behaviour for small $t$, as we consider in this paper, this ratio is numerically equal to the ratio $\sigma_{\mathrm{pp}}^{\mathrm{el}, \mathrm{I}} / \sigma$ between integrated elastic (from the imaginary part) and total pp cross section. Thus this elastic ratio is also nearly $1 / 3$, and the inelastic ratio is $\sigma_{\mathrm{pp}}^{\text {inel }} / \sigma \approx 2 / 3$ at the highest observed energies. Equation (8) indicates that this ratio converges to 0.3588 for $\sqrt{s} \rightarrow \infty$, which is far from the value $1 / 2$ that is characteristic of the idea of a black disk. Our results show that there is no such black disk behaviour [10]. This point will be discussed later in $b$-space representations.

We remark that we have used the slope $B_{I}$ in the ratio (7) defined above. We may similarly define the ratio using

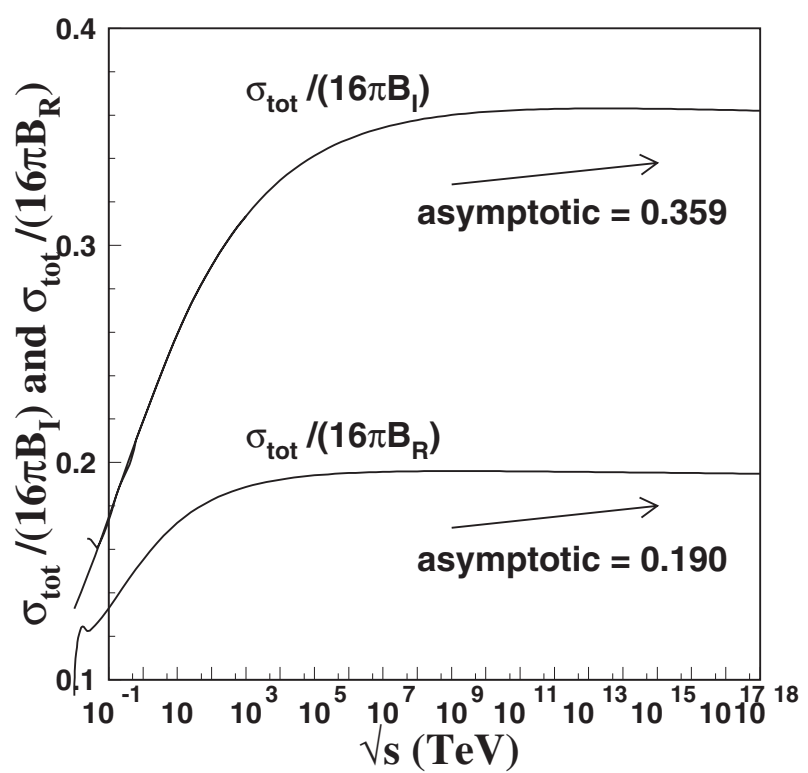

Figure 1. Energy dependence of the dimensionless ratios between total pp cross section and the slopes $B_{I}$ and $B_{R}$, as defined by Eqs. ((7), (9)). The expressions have finite asymptotic limits, as shown in equations and in the plots.

the $B_{R}$ slope, and then we obtain the high $\sqrt{s}$ behaviour

$$
R_{R}=\frac{1}{(\hbar c)^{2}} \frac{\sigma}{16 \pi B_{R}} \rightarrow 0.1896+\frac{0.325061}{\log \sqrt{s}}-\frac{5.23579}{\log ^{2} \sqrt{s}} .
$$

With pure exponential form in the real amplitude, this fraction would be equal to the ratio $\left(\sigma_{\mathrm{pp}}^{\mathrm{el}, \mathrm{R}} / \rho^{2}\right) / \sigma$. Since $\rho$ is small, the contribution of the real part to the integrated elastic cross section is also small.

The energy dependence of the two ratios $R_{I}$ and $R_{R}$ is shown ink Fig. 1.

\section{Galuber calculation}

The information on the parameters given above for the pp interaction enters in the calculation of production cross section $\sigma_{\mathrm{p} \text {-air }}^{\text {prod }}$ that is obtained from the analysis of EAS in the standard Glauber approximation [11-13].

In the approximation where the forward amplitudes are expressed as pure exponential form in $t$-space, the corresponding amplitudes in $b$-space become Gaussians in $b$,

$$
\begin{aligned}
\widehat{T}_{\mathrm{pp}}(s, \vec{b}) & =\widehat{T}_{R}(s, \vec{b})+i \widehat{T}_{I}(s, \vec{b}) \\
& \rightarrow \frac{\sigma_{\mathrm{pp}}^{\text {tot }}}{4 \pi(\hbar c)^{2}}\left[\frac{\rho}{B_{R}} e^{-\frac{b^{2}}{2 B_{R}}}+i \frac{1}{B_{I}} e^{-\frac{b^{2}}{2 B_{I}}}\right] .
\end{aligned}
$$

Here, our forward amplitudes $(s, t)$ show different $t$ behaviour in the imaginary and real parts, with different slopes $B_{I}$ and $B_{R}$. In terms of the eikonal function $\chi(s, \vec{b})$ this is written

$$
-i \widehat{T}_{\mathrm{pp}}(s, \vec{b})=1-e^{i \chi_{\mathrm{pp}}(s, \vec{b})} \equiv \Gamma_{\mathrm{pp}}(s, \vec{b}) .
$$



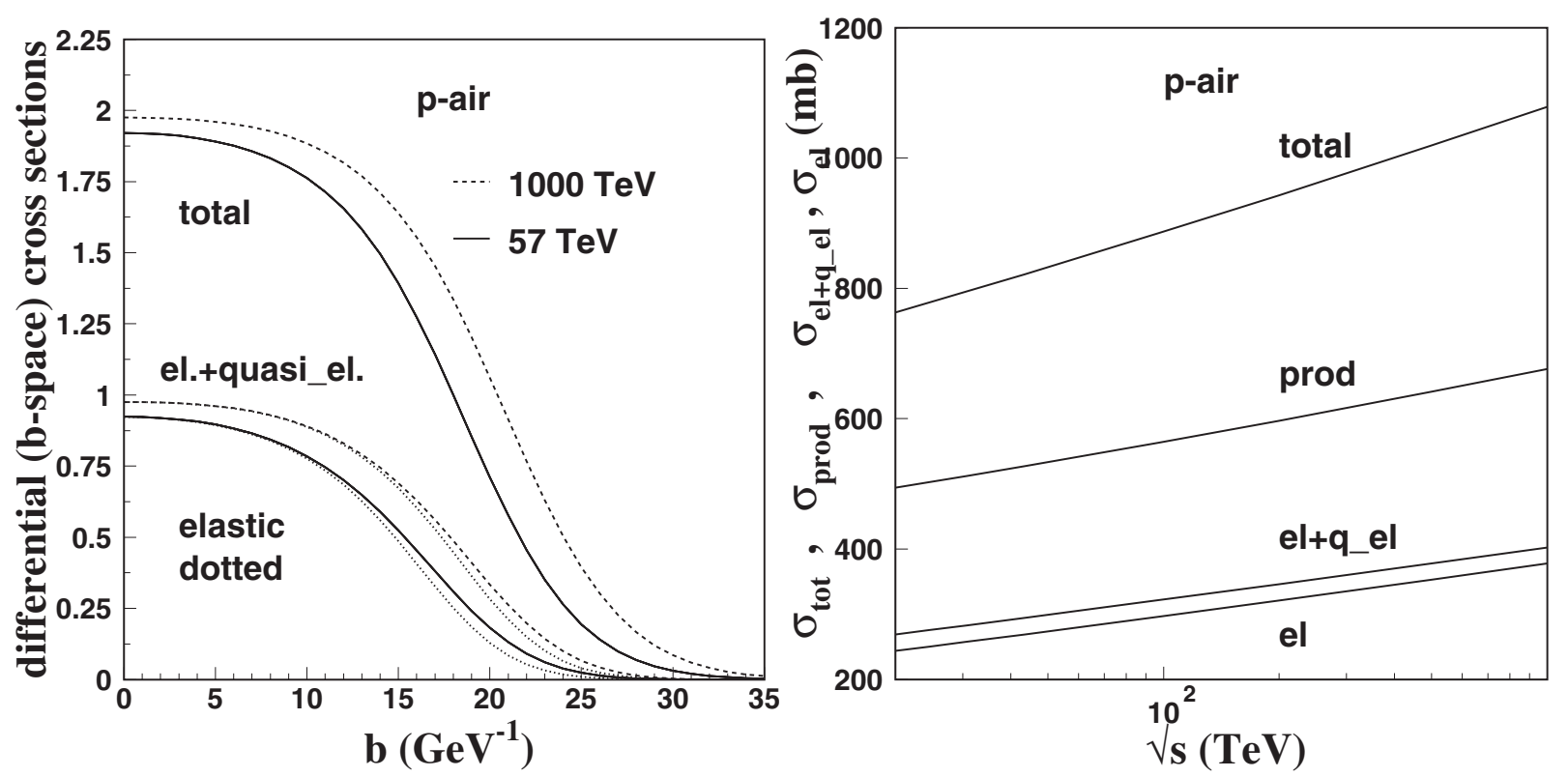

Figure 2. The quantities $d \sigma_{\mathrm{p}-\text { air }}^{\text {tot }} / d^{2} \vec{b}$ and $d \sigma_{\mathrm{p}-\text { air }}^{\mathrm{el}+\mathrm{q}-\mathrm{el}} / d^{2} \vec{b}$ are plotted as functions of the p-air impact parameter $b$ for the energies 57 and $1000 \mathrm{TeV}$. As the energy increases, the saturation limits 2 and 1 are approached by the total and inelastic parts for small $b$. The integrated quantities are shown in the second part of the figure. The difference between elastic+quasi-elastic and purely elastic terms is small.

The term $e^{i \chi_{\mathrm{pp}}(s, \vec{b})}$ represents the S-matrix function in $b$ space. The optical theorem for pp scattering appears as

$$
\sigma_{\mathrm{pp}}^{\text {tot }}(s)=2(\hbar c)^{2} \Re \int d^{2} \vec{b} \Gamma_{\mathrm{pp}}(s, \vec{b}) .
$$

Analogously, for elastic scattering in the p-A system, we define a quantity $\Gamma_{\mathrm{pA}}(s, \vec{b})$ that satisfies the optical theorem for the pA total cross section

$$
\sigma_{\mathrm{pA}}^{\mathrm{tot}}(s)=2(\hbar c)^{2} \Re \int d^{2} \vec{b} \Gamma_{\mathrm{pA}}(s, \vec{b}) .
$$

$\Gamma_{\mathrm{pA}}(s, \vec{b})$ is the reaction matrix element averaged over all target nucleon wavefunction inside the nucleus.

To describe the observed phenomena in EAS, we need to evaluate the quantity

$$
\sigma_{\mathrm{p}-\text { air }}^{\text {prod }}=\sigma_{\mathrm{p}-\text { air }}^{\text {tot }}-\left(\sigma_{\mathrm{p}-\text { air }}^{\mathrm{el}}+\sigma_{\mathrm{p}-\text { air }}^{\mathrm{q}-\mathrm{el}}\right)
$$

that is determined experimentally. The quantities named p-air are averages over a mixture of nitrogen and oxygen nuclei.

Stressing that we provide reliable information on cross sections and amplitude slopes for the pp scattering input, and a proper, although simple, treatment of Glauber framework, our calculations of $\sigma_{\mathrm{p} \text {-air }}^{\text {prod }}$ is adequate for the study of EAS data, as shown in the next section.

The dimensionless quantities that give the $b$-dependence of the total, elastic+quasi-elastic and pure elastic cross sections for the p-air system (taking averages over nitrogen and oxygen components)

$$
\frac{d \tilde{\sigma}_{\mathrm{p}-\mathrm{air}}^{\mathrm{tot}}}{d^{2} \vec{b}}(s, b), \frac{d \tilde{\sigma}_{\mathrm{p}-\text { air }}^{\mathrm{el}+\mathrm{q}-\mathrm{el}}}{d^{2} \vec{b}}(s, b), \frac{d \tilde{\sigma}_{\mathrm{p}-\mathrm{air}}^{\mathrm{el}}}{d^{2} \vec{b}}(s, b)
$$

are represented in Fig. 2 for the energies $\sqrt{s}=57$ and $\sqrt{s}=1000 \mathrm{TeV}$. As in the pp system, the total and inelastic cross sections for small $b$ approach the limits 2 and 1 as the energy increases. There is little difference between the elastic+quasi-elastic and the pure elastic quantities.

The integrated quantities $\sigma_{\mathrm{p}-\text { air }}^{\text {tot }}(s), \sigma_{\mathrm{p}-\text { air }}^{\mathrm{el}}+\sigma_{\mathrm{p}-\text { air }}^{\mathrm{q}-\mathrm{el}}(s)$ and $\sigma_{\mathrm{p}-\text { air }}^{\mathrm{el}}(s)$ are shown in the second part of the same figure. The ratio $\sigma_{\mathrm{p} \text {-air }}^{\text {el }} / \sigma_{\mathrm{p}-\text { air }}^{\text {tot }}$ is 0.33 at $57 \mathrm{TeV}$ and 0.35 at $1000 \mathrm{TeV}$. The difference between elastic+quasi-elastic and purely elastic contributions is remarkably small, of about 18 at $50 \mathrm{GeV}$ and falling steadily to zero as the energy increases. The inelastic p-air cross section is about $2 / 3$ of the total, as in the pp system.

\section{Comparison with data}

Figure 3 shows our calculation of $\sigma_{\mathrm{p}-\text { air }}^{\text {prod }}$ with a solid line, together with the data points from EAS experiments [15-22].

The procedure is straightforward and unique, without free parameters, made with inputs given by our model for the pp interaction that describes the elastic differential cross sections at all energies from $20 \mathrm{GeV}$ to $8 \mathrm{TeV}$ in the whole $t$-range. For the application in Glauber calculation of the p-air processes, the model enters only in its forward scattering limit, and is represented by Eqs. ((2)-(5)). Although a pure Gaussian in $b$-space is not adequate for the description of the $\mathrm{pp}$ amplitudes for large $|t|$, in the Glauber calculation the log-squared increases of $\sigma, B_{I}, B_{R}$ are consequence of the Yukawa-like behaviour of the exact amplitudes, which do not violate unitarity or dispersion relations [4]. Thus we consider that this is a reliable input.

The figure shows that in general there is good agreement between data and the calculation of $\sigma_{\mathrm{p}-\text { air }}^{\mathrm{prod}}$, without any systematic deviation that could require additional terms beyond the basic Glauber form. At high energies above $10 \mathrm{TeV}(\sqrt{s}$ in the proton-proton 


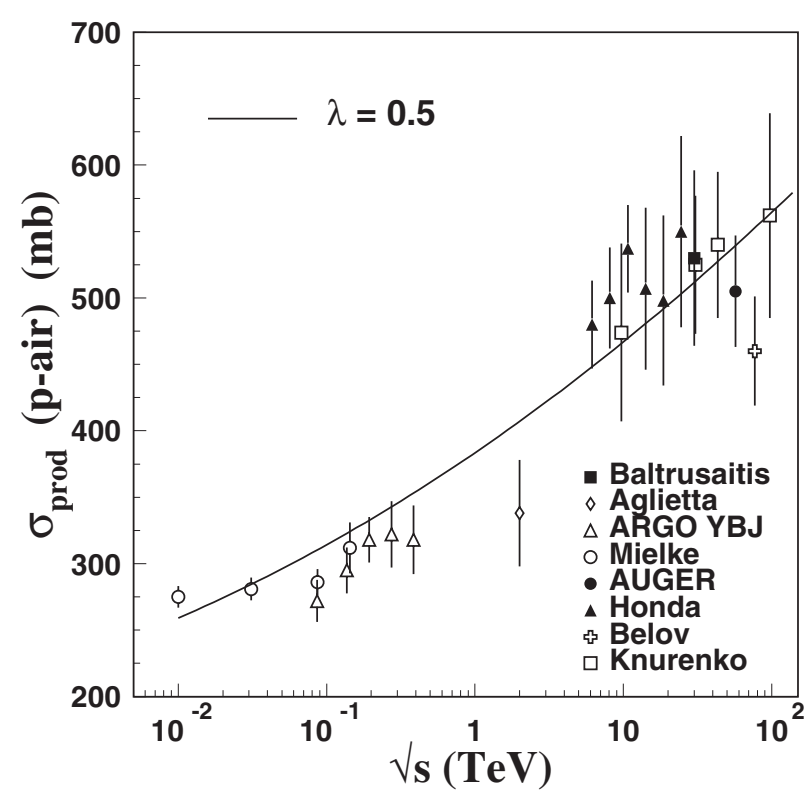

Figure 3. Our calculation of the p-air production cross section is represented by the solid line, that is well represented by Eq. (15). The data are from several experiments [15-22]. Both data and calculations increase with the energy with a $\log ^{2} \sqrt{s}$ form.

system) the agreement is particularly satisfactory within uncertainties of the present experimental information. In the low energy region we observe that data from the ARGO-YBJ experiment [20] is below the theoretical curve, while the data from the Kaskade experiment [21] do not shown the same systematic deviation.

The theoretical curve for the production cross section can be put in the simple and convenient form

$$
\sigma_{\mathrm{p}-\text { air }}^{\text {prod }}=383.474+33.158 \log \sqrt{s}+1.3363 \log ^{2} \sqrt{s},
$$

with $\sqrt{s}$ in TeV.

We observe that the data and our calculations of $\sigma_{\mathrm{p}-\text { air }}^{\text {prod }}$ increase with similar $\log ^{2} \sqrt{s}$ dependence as the pp cross sections, but more slowly. To compare the two rates and give more evidence of regularity in the data, we show in Fig. 4 the relation $\sigma_{\mathrm{p}-\text { air }}^{\text {prod }} / \sigma(\mathrm{pp})$ for a set of selected data (chosen by regularity reasons) together with our calculations. The ratio decreases regularly, approaching a finite and distant asymptotic limit, as pointed out by the relation of forms in Eqs. (15) and (2). The existence of a finite asymptotic limit for this ratio and its numerical value at ultra-high energies is important.

To exhibit more clearly the connection of this experimental behaviour with the geometric character of the $b$-distributions that build the cross sections values, we present in Fig. 5 the forms of the differential (in $b$-space) cross sections of the pp and p-air systems, at $\sqrt{s}=57$ $\mathrm{TeV}$. At this energy, we confirm that, although the Gaussian approximation fails for $\mathrm{pp}$ cross sections, for $\mathrm{p}$-air Glauber calculation the effect of using pure Gaussian instead of the full exact amplitudes is negligible. The distributions show smoothly decaying tails, and their integrations lead to ratios as $\sigma^{\mathrm{el}} / \sigma^{\text {tot }}(\mathrm{pp})=0.28, \sigma^{\mathrm{el}} / \sigma^{\text {tot }}(\mathrm{p}-$ air $)=0.33$,

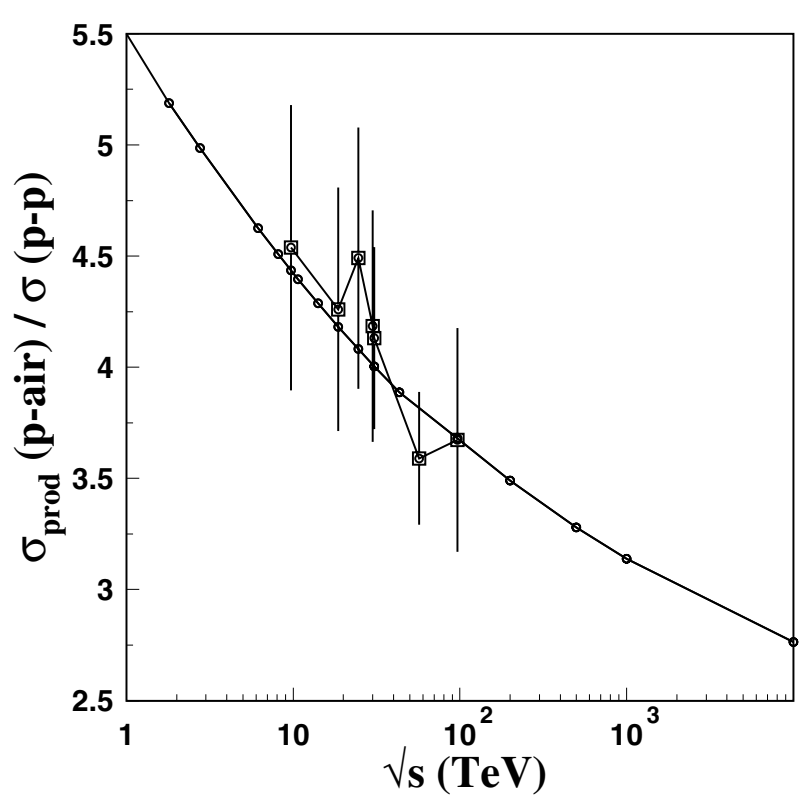

Figure 4. Ratio of p-air and pp cross sections. We show our calculation in solid line together with selected data [15,17-19]. We observe regular behaviour in the energy variation of the data for the ratio, that slowly approaches a finite asymptotic limit.

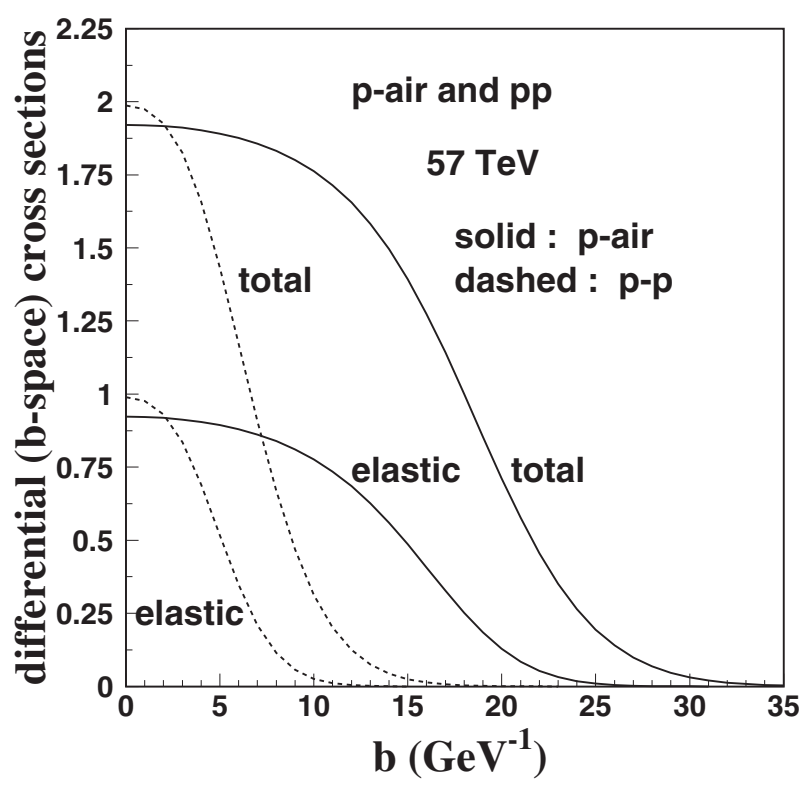

Figure 5. Differential cross sections of the form $d \tilde{\sigma} / d^{2} \vec{b}(s, b)$ (as in Eq. (14)) for the pp and p-air systems at $\sqrt{s}=57 \mathrm{TeV}$. The smoothly decaying tails show that neither the pp nor the p-air systems indicate a black-disk behaviour. This has consequences for the asymptotic behaviour. The calculation for $\mathrm{pp}$ is made with the full amplitudes of our model [1-5], according to Eq. (16).

$\sigma_{\mathrm{p}-\text { air }}^{\mathrm{prod}} / \sigma_{\mathrm{pp}}^{\text {tot }}=3.83$, that show that neither the pp nor the $\mathrm{p}$ air systems tend to a black-disk behaviour, and also that the integrated distributions are in agreement with the data in Fig. 4.

It seems that, up to this energy domain, the confrontation of our calculation with data at high energies does not indicate the need of contributions beyond the standard Glauber calculation. However, the EAS data are not regular with large error bars, due to uncertainties in 

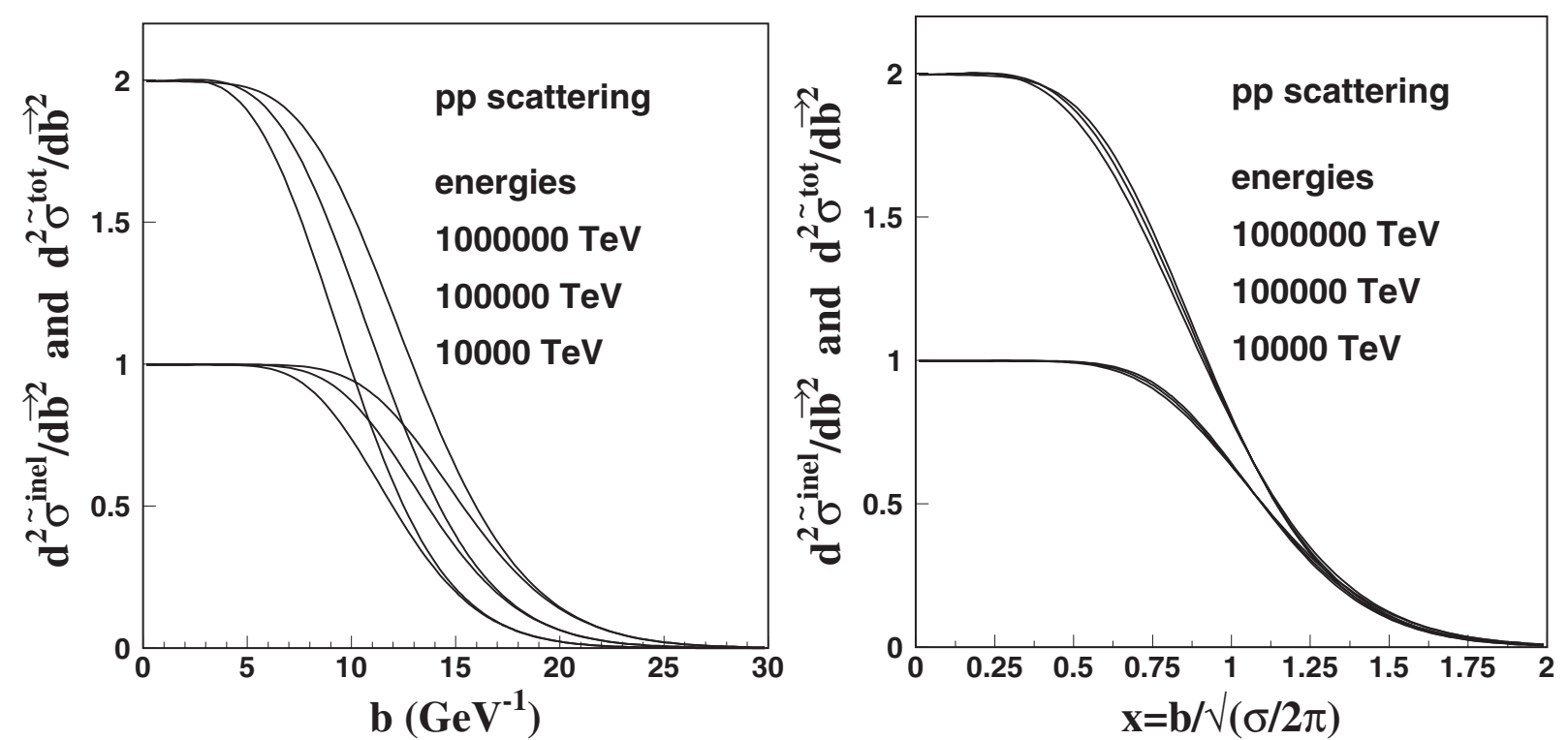

Figure 6. Dimensionless differential $b$-space cross sections for total and inelastic pp interactions. The plotted energies are $10^{4}, 10^{5}$ and $10^{6} \mathrm{TeV}$. In the second part of the figure, the cross sections are plotted against the scaled variable $x$, showing universal behaviour.

the extraction of values for $\sigma_{\mathrm{p}-\text { air }}^{\text {prod }}$ Improvement in the quality of future data may indicate influence of processes occurring in intermediate states of the p-air collision, as nucleon excitations, correlations, shadowing. A particular example is given by the recent AUGER measurement at $57 \mathrm{TeV}$, that seems to indicate a lower value with respect to the general trend of the data, although the error bar is quite large.

In the low energy region, in the data of the ARGO YBJ collaboration [20] there may be a regular deviation of our calculations. It may be that effects that are not observable at $100 \mathrm{TeV}$ may become important in this range. Anyhow, the discrepancies are not large, amounting to a maximum of $10 \%$ : at $\sqrt{s}=0.0865 \mathrm{TeV}$ the ARGO YBJ experiment gives $\sigma_{\mathrm{p} \text {-air }}^{\text {prod }}=272 \pm 15.8 \mathrm{mb}$, while the theory gives $307.21 \mathrm{mb}$. On the contrary, at $\sqrt{s}=0.031 \mathrm{TeV}$ the Kaskade experiment [21] and the theoretical value coincide very well (at $281 \pm 8.5$ and $286 \mathrm{mb}$ respectively).

In general, there seems to be more room for improvement in the measurements than in the theoretical calculation, and we believe that our pp input together with the basic Glauber calculation have successfully passed the test in the comparison with EAS data.

\section{Impact parameter representation}

Although the $b$-space representation does not exactly mean the classical impact parameter space, and is not observable, it gives us a geometrical image of the behaviour of a proton from the amplitude. In the forward amplitude, the corresponding $b$-space is a Gaussian form of the eikonal thickness function. The complete form of Eq. (16) for the pp scattering amplitude in $b$-space is

$$
\begin{aligned}
& \hat{T}_{K}(s, \vec{b})=\frac{\alpha_{K}}{2 \beta_{K}} e^{-b^{2} / 4 \beta_{K}} \\
& +\lambda_{K} \frac{2 e^{\gamma_{K}-\sqrt{\gamma_{K}^{2}+b^{2} / a_{0}}}}{a_{0} \sqrt{\gamma_{K}^{2}+b^{2} / a_{0}}}\left[1-e^{\gamma_{K}-\sqrt{\gamma_{K}^{2}+b^{2} / a_{0}}}\right],
\end{aligned}
$$

where $K=R, I$ indicates either the real or the imaginary part and $a_{0}=1.39 \mathrm{GeV}^{-2}$ is a constant from the stochastic vacuum model $[6,7]$. The second term comes from the stochastic vacuum model, representing the effect of interaction of Wilson loops. Note that due to the presence of this term, the first Gaussian term does not coincide with that used in the previous section. That is, the slope parameters $B_{R, I}$ are not given by $\beta_{K}$ 's above but defined as $B_{K} \equiv d \ln T_{K}^{2} / d t$ which contains contributions from the two terms. Furthermore, the large $b$ behaviour of the amplitudes are not Gaussian anymore, but falls down with a Yukawa-like tail, $\sim e^{-b / b_{0}} / b$. We observe also a very nice geometrical scaling property in differential cross sections in $b$-space. At asymptotic high energies, they scale with the variable $x=b / \sqrt{\sigma_{T}(\sqrt{s})}$, as shown in Fig. 6. As seen clearly from this figure, the profile functions (differential cross sections in $b$-space) as function of $x$ degenerate for all high energies to respective unique distribution, and their forms are much different from that expected for the black disk (sharp edged Heaviside step function). In fact we can show that this diffused nature of the proton profile functions is responsible for the deviations of the ratios $R_{I}$ $\sigma_{\mathrm{pp}}^{\mathrm{el}, \mathrm{I}} / \sigma$ and $\sigma_{\mathrm{pp}}^{\text {inel }} / \sigma$ from the black disk value $1 / 2$ [3].

It is important to note that the Gaussian approximation for $\mathrm{pp}$ amplitudes at these energies (including that of Fig. 5) violates unitarity so that these curves are calculated with the full exact amplitude, Eq. (16). The non-Gaussian behaviour of the shape function has been pointed out also in $[27,28]$.

\section{Final remarks and comments}

The amplitudes that we have constructed to describe accurately the pp elastic differential cross sections at energies from $20 \mathrm{GeV}$ to $8 \mathrm{TeV}$ are used in Glauber formalism to evaluate the p-air production cross section in the energy domain of EAS/CR experiments. Our prediction for the whole energy interval from $10 \mathrm{GeV}$ to 


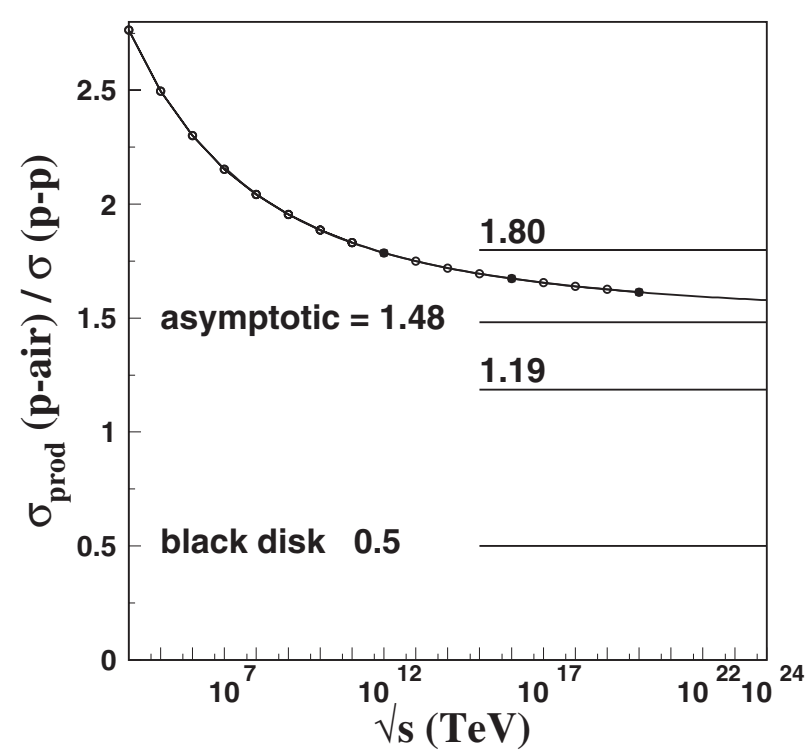

Figure 7. Ratio of p-air and pp cross sections at ultra-high energies. Calculations are marked with dots and connected with a continuous line. The dashed line is given analytically by the fraction of $\log ^{2}$ forms for $\sigma_{\mathrm{p}-\text { air }}^{\text {inel }}(s)$ and $\sigma_{\mathrm{pp}}^{\text {tot }}(s)$, given in the text. It gives good representation of the points for energies above $10^{6} \mathrm{TeV}$ and tends to the asymptotic limit 1.48 . As we see from this figure, the asymptotic value is attained only for really large $\sqrt{s}$, say $\sqrt{s} \gg 10^{20} \mathrm{TeV}$.

$100 \mathrm{TeV}$ of p-air production cross section is shown in Fig. 3.

The calculations with Glauber approach depend crucially on the input values of $\sigma_{\mathrm{pp}}^{\text {tot }}(s)$ and $B_{I}(s)$, and thus the results obtained for the high energies of the $\mathrm{CR}$ experiments are important tests of the energy dependences that we propose for these quantities, given in Eqs. ((2), (3)). It is particularly remarkable that the $\log ^{2}$ dependence that we propose for $B_{I}(s)$ predicts higher values for the extrapolated values of this quantity, and the data seem to be consistent with this. Thus at $57 \mathrm{TeV}$ we have $B_{I}=$ $25 \mathrm{GeV}^{-2}$, value that is higher than the usual obtained, for example from Donnachie-Landshoff or Regge form. The comparison with CR data helps to test such alternatives.

The extraction of fundamental information on the energy dependence of pp total cross section from CR/EAS measurements depends on this point. Thus our prediction for $\mathrm{pp}$ cross section at $57 \mathrm{TeV}$ is $\sigma_{p p}=140.7 \mathrm{mb}$. In the experimental paper [15], where the measured value for $\sigma_{\mathrm{p} \text {-air }}^{\text {prod }}$ is below our calculation (see Fig. 3), and other theoretical models for $\sigma(s)$ and $B_{I}(s)$ are used, the reported value for $\sigma$ is $\sigma(\mathrm{pp})=133 \pm 29 \mathrm{mb}$. Hopefully this important question will be investigated in future CR measurements.

From our representation of the scattering amplitudes we can calculate the asymptotic values of quantities that approach finite values at high energies. These values are important for the geometric interpretation of the dynamics, as can be studied in the representation of the impact parameter $b$. For example, the behaviour of the ratios $\sigma_{\mathrm{pp}}^{\text {tot }} / B_{I}$ and $\sigma_{\mathrm{pp}}^{\text {tot }} / B_{R}$ are connected with integrated elastic $\mathrm{pp}$ cross sections and thus with the rate of inelastic processes at high energies in the pp system. Our results show that $\sigma_{\mathrm{pp}}^{\text {inel }} / \sigma_{\mathrm{pp}}^{\text {tot }} \sim \approx 2 / 3$ at very high energies. This ratio and $R_{I}$ together show that the geometric nature of pp cross section is far the black disk form. This is so even asymptotically.

To acquire a better feeling about the regularity of the energy dependence of the data and its representation by the theoretical calculation, we present in Fig. 7 results on the ratio between $\mathrm{p}$-air and pp cross sections. The figure shows that this ratio has the important property of approaching a finite value for infinite energy. This information if of fundamental importance for the understanding of the geometric nature of the $\mathrm{pp}$ interaction and its energy dependence. We show that this ratio is intimately related with the relation $\sigma$ (ppinelastic) $/ \sigma$ (pptotal) and with the behaviour of the eikonal functions for large $b$.

The important question of the energy dependence of the ratio of p-air to pp cross sections is studied in a direct way, using properties of the $b$ dependence of pp interaction at high energies. We show that the Yukawa-like behaviour of the interaction range, inspired in the stochastic vacuum model, explains quantitatively the value of the asymptotic limit of the ratio $\sigma_{\mathrm{p}-\text { air }}^{\text {ine }} / \sigma_{\mathrm{pp}}^{\text {tot }}$.

In this work, we have applied the standard Glauber model, considering that the nucleons are the scattering centers. Of course, for a ultra-high energy domain, where the pp cross section becomes comparable to the geometric cross section of a target nucleus, the Glauber approach itself may be questionable. In the Glauber approach of pA cross section, the scattering centers inside the target are nucleons, with a fixed distribution determined by the nuclear wave function. However, at the energies where the interaction size of pp becomes large enough so that their superposition becomes not negligible, the scattering centers are rather partons and not nucleons. Then the energy dependence of $\mathrm{pA}$ cross section can become drastically different [29]. Here we have an open question. Further theoretical investigations of microscopic structures leading to the asymptotic behaviour in p-air cross sections will be very interesting.

The authors wish to thank the Brazilian agencies CNPq, CAPES, PRONEX and FAPERJ for financial support.

\section{References}

[1] A. Kendi Kohara, E. Ferreira and T. Kodama, Eur. Phys. J. C, 73, 2326 (2013)

[2] A. K. Kohara, E. Ferreira and T. Kodama, Phys. Rev. D 87, 054024 (2013)

[3] A. K. Kohara, E. Ferreira and T. Kodama, J. Phys. G: Nucl. Part. Phys. 41 (2014) 115003

[4] A. K. Kohara, E. Ferreira and T. Kodama, "Energy Dependence and Asymptotic Behaviour of $p p$ scattering Amplitudes", to be published (2014)

[5] A. Kendi Kohara, E. Ferreira and T. Kodama, arXiv: 1408.1599 [hep-ph]

[6] H.G. Dosch, Phys. Lett. B 190, 177 (1987); H.G. Dosch, E. Ferreira, A. Kramer Phys. Rev. D 50, 1992 (1994) 
[7] E. Ferreira and F. Pereira, Phys. Rev. D 59, 014008 (1998); Phys. Rev. D 61, 077507 (2000)

[8] J. Beringer et al. (Particle Data Group), Phys. Rev. D 86, 010001 (2012)

[9] E. Ferreira, Int. Jour. Mod. Phys. E 16, 2893 (2007)

[10] D.A. Fagundes, M.J. Menon, P.V.R.G. Silva, J. Phys. G40, 065005 (2013)

[11] R.J. Glauber, Phys. Rev. 100, 242-248 (1955); R.J. Glauber and G. Matthiae, Nucl. Phys. B 21, 135-157 (1970)

[12] M. L. Good and W. D. Walker, Phys. Rev. 120, 18571860 (1960)

[13] R. Engel and R. Ulrich, Internal Pierre Auger Note GAP-2012, March 2012

[14] R. C. Barret and D. F. Jackson, Nuclear Size and Structure, Clarendon Oxford 1977

[15] P. Abreu et al, Auger Coll., Phys. Rev. Lett. 109, 062002 (2012)

[16] K. Belov et al., HiRes Coll., Fly's Eye Exp., Nucl. Phys. B (Proc. Suppl) 151 (2006) 197-204

[17] R. M. Baltrusaitis, et al.,Fly's Eye Experiment, Phys. Rev. Lett. 52 (1984) 1380-1383

[18] M. Honda, Akeno Coll., Phys. Rev. Lett. 70 (1993) 525-528

[19] S. P. Knurenko et al., Yakutsk Array Coll., (1999), Proc. of 26th ICRC (ICRC 99) (Salt Lake City, USA) Vol 1, p. 372
[20] G. Aielli et al., ARGO-YBJ experiment, Phys. ReV. D 80, 092004 (2009)

[21] H. H. Mielke et al., Jour. Phys. G 20 (1994) 637

[22] M. Aglietta et al., Phys. Rev. D 79, 032004 (2009)

[23] T.K. Gaisser, U.P.Sukhatme and G.B. Yodh, Phys. Rev.D 36 (1987) 1350-1357

[24] B.Z. Kopeliovich, N.N. Nikolaev, I.K. Potashnikova Phys. Rev. D 39 (1989) 769

[25] R. Engel, T.K. Gaisser, P. Lipari and T. Stanev, Phys. Rev. D 58 (1998) 014019

[26] J. Dias de Deus, Nucl. Phys. B 59 (1973) 231; A.J. Buras, J. Dias de Deus, Nucl.Phys. B 71 (1974) 481; J. Dias de Deus, P. Kroll, J. Phys. G 9 (1983) L81; J. Dias de Deus, Acta Phys. Polon. B 6 (1975) 613

[27] T. Csorgo, R. J. Glauber, F. Nemes, arXiv: 1311. 2308v1 [hep-ph] (2013)

[28] F. Nemes and T. Csőgő, arXiv: 1204.5617v2 [hepph] (2012); T. Csőgő, talk presented at International Workshop on Collectivity in Relativistic Heavy Ion Collisions, Kolymbari, Crete, Greece, Sept 14-20, 2014

[29] L. Portugal and T. Kodama, Nuclear Physics A 837 (1), 1-14 (2010)

[30] D.A. Fagundes, A. Grau, G. Pancheri, Y. N. Srivastava and O. Shekhovtsova, arXiv: 1408.2921 [hep-ph] 